\title{
MINIMAL MARTINGALE MEASURE ON A FINITE PROBABILITY SPACE
}

UDC 519.21

\author{
VADYM DOROSHENKO
}

\begin{abstract}
Necessary and sufficient conditions are established for the existence of a minimal martingale measure for a discrete time financial market on a finite probability space.
\end{abstract}

\section{INTRODUCTION}

The definition of the minimal martingale measure is introduced in the papers [1] and 2]. The minimal martingale measure is used as an auxiliary technical tool in the problem of the local minimization of the risk in [1] and 2]. The definition of the minimal martingale measure used in this paper is given in [3].

Minimal martingale measures are used in models with continuous time, too. Some of the properties of minimal martingale measures are considered in [4]-7]; applications in hedging are described in [1, 8, and those in pricing the contingent claims are given in 9, 10.

In the current paper, we consider the question of the existence and evaluation of the minimal martingale measure for a financial market defined on a finite probability space. We develop a procedure to evaluate a minimal martingale measure. Examples of evaluation of minimal martingale measures are also given.

The paper is organized as follows. Section 1 introduces some necessary notation. Section 2 considers a one-period model of the financial market. Necessary and sufficient conditions for the existence of a minimal martingale measure are given in Theorem 2.1. Section 3 considers a multiperiod model of the financial market. We propose a procedure that determines whether or not a minimal martingale measure exists and allows one to evaluate it in the case where such a measure exists. Section 4 contains some examples of the evaluation of minimal martingale measures by using the procedure developed in Section 3 .

\section{Definitions}

Consider a model of the financial market with discrete time and $d+1$ assets. We assume that one of these assets is risk-free, while the others $d$ assets are risky. Let $(\Omega, \mathcal{F}, \mathrm{P})$ be a probability space and let $\{\varnothing, X\}=\mathcal{F}_{0} \subset \mathcal{F}_{1} \subset \cdots \subset \mathcal{F}_{T}=\mathcal{F}$ be a filtration of $\sigma$-algebras in this space. The asset prices at moments $t=0, \ldots, T$ are given by

$$
S_{t}=\left(S_{t}^{0}, S_{t}^{1}, \ldots, S_{t}^{d}\right),
$$

where $S_{t}^{0}>0$ is nonrandom and the $S_{t}^{k}$ are $\mathcal{F}_{t}$-measurable functions. 
The symbol $\mathbb{T}$ denotes the set $\{0, \ldots, T\}$.

Definition 1.1. The processes

$$
X_{t}^{i}=\frac{S_{t}^{i}}{S_{t}^{0}}, \quad t \in \mathbb{T}, i=1, \ldots, d,
$$

are called the discounted price processes.

Denote by $X^{k}=\left\{X_{t}^{k}, t \in \mathbb{T}\right\}$ the price process for the $k$-th asset.

Definition 1.2. A stochastic process $\left(U_{t}\right)_{t \in \mathbb{T}}$ defined on a probability space $(\Omega, \mathcal{F}, \mathrm{P})$ is called adapted with respect to a filtration $\mathcal{F}_{0} \subset \mathcal{F}_{1} \subset \cdots \subset \mathcal{F}_{T}$ if the random variable $U_{t}$ is $\mathcal{F}_{t}$-measurable for all $t=0, \ldots, T$.

Definition 1.3. A measure $\widetilde{P}$ is called a martingale measure if it is equivalent to $P$ and if all price processes $X^{k}, k=1, \ldots, N$, are martingales with respect to $\widetilde{\mathrm{P}}$.

Definition 1.4. Let $\mathcal{F}_{0} \subset \mathcal{F}_{1} \subset \cdots \subset \mathcal{F}_{T}$ be a filtration of $\sigma$-algebras defined on a probability space $(\Omega, \mathcal{F}, \mathrm{P})$. Two processes $U=\left(U_{t}\right)_{t \in \mathbb{T}}$ and $V=\left(V_{t}\right)_{t \in \mathbb{T}}$ are called strongly orthogonal if they are adapted with respect to this filtration and if

$$
\operatorname{cov}\left(\left(U_{t+1}-U_{t}\right)\left(V_{t+1}-V_{t}\right) \mid \mathcal{F}_{t}\right)=0 \quad(\bmod \mathrm{P}), \quad t=0, \ldots, T-1 .
$$

We write $U \perp V$ if two processes $U$ and $V$ are strongly orthogonal.

Note that if one of the adapted processes $\left(U_{t}\right)_{t \in \mathbb{T}}$ or $\left(V_{t}\right)_{t \in \mathbb{T}}$ in Definition 1.4 is a martingale, then

$$
\operatorname{cov}\left(\left(U_{t+1}-U_{t}\right)\left(V_{t+1}-V_{t}\right) \mid \mathcal{F}_{t}\right)=\mathrm{E}\left(\left(U_{t+1}-U_{t}\right)\left(V_{t+1}-V_{t}\right) \mid \mathcal{F}_{t}\right) .
$$

We also say that two processes are P-strongly orthogonal to indicate the measure used to evaluate expectations in Definition 1.4.

Definition 1.5. A measure $Q$ on $(\Omega, \mathcal{F})$ is called a minimal martingale measure if

(1) $\mathrm{Q}$ is a martingale probability measure that is equivalent to $\mathrm{P}$;

(2) $\mathrm{E}(d Q / d \mathrm{P})^{2}<\infty$;

(3) each square integrable $\mathrm{P}$-martingale that is strongly orthogonal to $X^{i}, i=$ $1, \ldots, d$, is a $\mathrm{Q}$-martingale.

In what follows we consider a finite probability space

$$
\Omega=\left\{\omega_{1}, \ldots, \omega_{N}\right\}, \quad \mathcal{F}=2^{\Omega},
$$

where $N \geq 1$ is a fixed number. We assume that $\mathrm{P}\left(\left\{\omega_{i}\right\}\right)>0, i=1, \ldots, N$.

The aim of this paper is to find necessary and sufficient conditions for the existence of a minimal martingale measure in the case where a financial market is defined on a finite probability space.

Since the probability space is assumed to be finite, all probabilities as well as random variables defined on it can naturally be identified with vectors of the Euclidean space $\mathbb{R}^{N}$. Let $(a, b)$ be the standard scalar product of two vectors in $\mathbb{R}^{N}$, namely

$$
(a, b)=\sum_{k=1}^{N} a_{k} b_{k},
$$

where $a=\left(a_{1}, \ldots, a_{N}\right)$ and $b=\left(b_{1}, \ldots, b_{N}\right) \in \mathbb{R}^{N}$.

If $a=\left(a_{1}, \ldots, a_{N}\right)$ and $b=\left(b_{1}, \ldots, b_{N}\right) \in \mathbb{R}^{N}$, then $a b$ denotes the vector

$$
a b=\left(a_{1} b_{1}, \ldots, a_{N} b_{N}\right) .
$$




\section{ONE-PERIOD MODEL OF THE FINANCIAL MARKET}

First we consider a one-period model of the financial market, that is, the case of $T=1$. Let $Y^{i}=X_{1}^{i}-X_{0}^{i}, i=1, \ldots, d$, denote the gains. Put $p_{i}=\mathrm{P}\left(\left\{\omega_{i}\right\}\right), q_{i}=\mathrm{Q}\left(\left\{\omega_{i}\right\}\right)$, and $y_{j i}=Y^{j}\left(\omega_{i}\right), i=1, \ldots, N, j=1, \ldots, d$.

Lemma 2.1. A minimal martingale measure $\mathrm{Q}$ is a linear combination of $\mathrm{P}$ and $\mathrm{P} Y^{1}$, $\ldots, \mathrm{P} Y^{d}$.

Proof. Let $M=\left(M_{0}, M_{1}\right)$ be a P-martingale that is orthogonal to the price processes $X^{i}=\left(X_{0}^{i}, X_{1}^{i}\right), i=1, \ldots, d$. Put $m_{k}=M_{1}\left(\omega_{k}\right), k=1, \ldots, N, m=M_{0}$.

The property that $M$ is a $\mathrm{P}$-martingale can be written as follows:

$$
\mathrm{E}_{\mathrm{P}}\left(M_{1}-M_{0}\right)=\sum_{k=1}^{N} p_{k}\left(m_{k}-m\right)=\left(\mathrm{P}, M_{1}-M_{0}\right)=0 .
$$

Thus $\mathrm{P} \perp\left(M_{1}-M_{0}\right)$.

Since $M$ is strongly orthogonal to $\left(X_{0}^{i}, X_{1}^{i}\right), i=1, \ldots, N$, we deduce that

$$
\mathrm{E}_{\mathrm{P}}\left(M_{1}-M_{0}\right)\left(X_{1}^{i}-X_{0}^{i}\right)=\mathrm{E}_{\mathrm{P}}\left(M_{1}-M_{0}\right) Y^{i}=\sum_{k=1}^{N} p_{k} y_{i k}\left(m_{k}-m\right)=0,
$$

that is, $\mathrm{P} Y^{i} \perp\left(M_{1}-M_{0}\right)$. Since $M$ is a $\mathrm{Q}$-martingale, we have $\mathrm{Q} \perp\left(M_{1}-M_{0}\right)$.

If $M_{1} \in\left(\left\langle\mathrm{P}, \mathrm{P} Y^{1}, \ldots, \mathrm{P} Y^{d}\right\rangle\right)^{\perp}$ is an arbitrary vector, then the stochastic process $M=$ $\left(0, M_{1}\right)$ is a $\mathrm{P}$-martingale, whence

$$
\mathrm{Q} \in\left(\left\langle\mathrm{P}, \mathrm{P} Y^{1}, \ldots, \mathrm{P} Y^{d}\right\rangle\right)^{\perp \perp}=\left\langle\mathrm{P}, \mathrm{P} Y^{1}, \ldots, \mathrm{P} Y^{d}\right\rangle .
$$

According to Lemma 2.1, there are $\alpha_{1}, \ldots, \alpha_{d} \in \mathbb{R}$ and $\beta \in \mathbb{R}$ such that

$$
\mathrm{Q}=\sum_{k=1}^{d} \alpha_{k} \mathrm{P} Y^{k}-\beta \mathrm{P}
$$

If the family of vectors $Y^{i}, i=1, \ldots, d$, is linearly dependent, then we consider its maximal linearly independent subfamily. In what follows we assume that the family of vectors $Y^{i}, i=1, \ldots, d$, is linearly independent.

Since $\mathrm{Q}$ is a martingale measure, $\left(\mathrm{Q}, Y^{i}\right)=0, i=1, \ldots, d$. This property can be written in the form of a system of $d$ equations and $d+1$ unknowns, namely

$$
\begin{gathered}
\alpha_{1}\left(\mathrm{P} Y^{1}, Y^{1}\right)+\cdots+\alpha_{d}\left(\mathrm{P} Y^{d}, Y^{1}\right)=\beta\left(\mathrm{P}, Y^{1}\right), \\
\cdots \\
\alpha_{1}\left(\mathrm{P} Y^{1}, Y^{d}\right)+\cdots+\alpha_{d}\left(\mathrm{P} Y^{d}, Y^{d}\right)=\beta\left(\mathrm{P}, Y^{d}\right) .
\end{gathered}
$$

Now we introduce a scalar product $[U, V]$ in $\mathbb{R}^{N}$ by

$$
[U, V]=(\mathrm{P} U, V)
$$

where $U=\left(u_{1}, \ldots, u_{N}\right)$ and $V=\left(v_{1}, \ldots, v_{N}\right)$. It is not complicated to check that $[U, V]$ is a scalar product, indeed.

Then the left hand side of the system (2) is the Gram matrix with respect to the scalar product $[\cdot, \cdot]$. Since the family of vectors $Y^{1}, \ldots, Y^{d}$ is linearly independent, the matrix of system (2) is nonsingular. Therefore, for each fixed $\beta$, there exists a unique solution of system (2). All the solutions of system (2) are proportional. Since $\mathrm{Q}=\left(q_{1}, \ldots, q_{N}\right)$ is a probability measure, $\sum_{k=1}^{N} q_{k}=1$. Hence only one of the proportional solutions determines a probability measure.

The following result is known in the general theory of minimal martingale measures (see [11]). 
Proposition 1. There exists at most one minimal martingale measure in a one-period model of the financial market.

Proof. Assume that there are two minimal martingale measures. Then the system of equations (2) has at least two solutions for an arbitrary maximal linearly independent subfamily of the system of vectors $Y^{i}, i=1, \ldots, d$. This is impossible and Proposition 1 is proved by contradiction.

Now we are ready to provide necessary and sufficient conditions for the existence of a minimal martingale measure.

Since $\left(\mathrm{P} Y^{i}, Y^{j}\right)=\mathrm{E}_{\mathrm{P}} Y_{i} Y_{j}$ and $\left(\mathrm{P}, Y^{i}\right)=\mathrm{E}_{\mathrm{P}} Y_{i}, i, j=1, \ldots, d$, one can rewrite system (2) as follows:

$$
\begin{gathered}
\alpha_{1} \mathrm{E}_{\mathrm{P}}\left(Y_{1}\right)^{2}+\cdots+\alpha_{d} \mathrm{E}_{\mathrm{P}} Y_{d} Y_{1}=\beta \mathrm{E}_{\mathrm{P}} Y_{1}, \\
\cdots \\
\alpha_{1} \mathrm{E}_{\mathrm{P}} Y_{d} Y_{1}+\cdots+\alpha_{d} \mathrm{E}_{\mathrm{P}}\left(Y_{d}\right)^{2}=\beta \mathrm{E}_{\mathrm{P}} Y^{d} .
\end{gathered}
$$

A minimal martingale measure exists if and only if system (3) has a solution

$$
\left(\alpha_{1}, \ldots, \alpha_{n}, \beta\right)
$$

such that $\mathrm{Q}=\sum_{k=1}^{d} \alpha_{k} \mathrm{P} Y^{k}-\beta \mathrm{P}$ is a probability vector with nonzero coordinates.

Put

$$
\begin{gathered}
\Delta=\operatorname{det}\left(\begin{array}{ccc}
\mathrm{E}_{\mathrm{P}}\left(Y^{1}\right)^{2} & \ldots & \mathrm{E}_{\mathrm{P}} Y^{1} Y^{d} \\
\ldots & & \\
\mathrm{E}_{\mathrm{P}} Y^{d} Y^{1} & \ldots & \left.\mathrm{E}_{\mathrm{P}}\left(Y^{d}\right)^{2}\right)
\end{array}\right), \\
\Delta_{i}=\operatorname{det}\left(\begin{array}{ccccc}
\mathrm{E}_{\mathrm{P}}\left(Y^{1}\right)^{2} & \ldots & \mathrm{E}_{\mathrm{P}} Y^{1} & \ldots & \mathrm{E}_{\mathrm{P}} Y^{1} Y^{d} \\
\ldots & & & & \\
\mathrm{E}_{\mathrm{P}} Y^{d} Y^{1} & \ldots & \mathrm{E}_{\mathrm{P}} Y^{d} & \ldots & \mathrm{E}_{\mathrm{P}}\left(Y^{d}\right)^{2}
\end{array}\right),
\end{gathered}
$$

where the numbers $\mathrm{E}_{\mathrm{P}} Y^{j}, j=1, \ldots, d$, form the $i$-th column in $\Delta_{i}$.

According to Cramer's rule,

$$
\alpha_{i}=\beta \frac{\Delta_{i}}{\Delta}, \quad i=1, \ldots, d .
$$

Thus

$$
\mathrm{Q}=\beta \sum_{k=1}^{d} \frac{\Delta_{k}}{\Delta} \mathrm{P} Y^{k}-\beta \mathrm{P}=-\beta\left(\mathrm{P}-\sum_{k=1}^{d} \frac{\Delta_{k}}{\Delta} \mathrm{P} Y^{k}\right) .
$$

Note that if a vector $\mathrm{Q}$ corresponds to a probability measure, then $q_{i}>0, i=1, \ldots, N$, and thus the following result holds.

Theorem 2.1. A minimal martingale measure exists if and only if all the numbers

$$
r_{i}=1-\sum_{k=1}^{d} \frac{\Delta_{k}}{\Delta} y_{k i}, \quad i=1, \ldots, d,
$$

are of the same sign.

Lemma 2.2. Let $d=1$. A minimal martingale measure exists if and only if

$$
y_{1 i} \mathrm{E}_{\mathrm{P}} Y^{1}<\mathrm{E}_{\mathrm{P}}\left(Y^{1}\right)^{2}, \quad i=1, \ldots, N .
$$


Proof. Since $d=1$, we have

$$
\Delta=\left(\mathrm{P} Y^{1}, Y^{1}\right)=\mathrm{E}_{\mathrm{P}}\left(Y^{1}\right)^{2}, \quad \Delta_{1}=\left(\mathrm{P}, Y^{1}\right)=\mathrm{E}_{\mathrm{P}} Y^{1} .
$$

Theorem 2.1 implies that a minimal martingale measure exists if and only if all the numbers $r_{i}=\mathrm{E}_{\mathrm{P}}\left(Y^{1}\right)^{2}-\mathrm{E}_{\mathrm{P}} Y^{1} y_{1 i}, i=1, \ldots, N$, are of the same sign. Since

$$
\sum_{i=1}^{N} p_{i} a_{i}=\mathrm{E}_{\mathrm{P}}\left(Y^{1}\right)^{2}-\left(\mathrm{E}_{\mathrm{P}} Y^{1}\right)^{2}>0,
$$

we conclude that all the $a_{i}$ cannot be negative. Therefore a minimal martingale measure exists if and only if all numbers $r_{i}$ are positive. This completes the proof of the lemma.

\section{MUltiperiod MODEL OF THE FINANCIAL MARKET}

Now we treat the case of $T>1$.

Let $\mathrm{Q}$ be a probability measure on the space $(\Omega, \mathcal{F}, \mathrm{P})$ equipped with a filtration $\{\varnothing, \Omega\}=\mathcal{F}_{0} \subset \mathcal{F}_{1} \subset \cdots \subset \mathcal{F}_{T}=\mathcal{F}$. Assume that $\mathrm{Q}$ is equivalent to the measure $\mathrm{P}$. Our aim is to find a condition imposed on $Q$ ensuring that $Q$ is a minimal martingale measure.

Let $R$ be an arbitrary probability measure on $(\Omega, \mathcal{F})$ that is equivalent to the measure P. For all $t \in \mathbb{T}$, denote by $R_{t}$ the restriction of the measure $R$ to the $\sigma$-algebra $\mathcal{F}_{t}$. Then $R_{0}(\varnothing)=0, R_{0}(\Omega)=1$, and the measure $R_{T}$ coincides with the measure $R$.

Clearly,

$$
\mathrm{E}_{R_{t}}\left(\xi \mid \mathcal{F}_{s}\right)=\mathrm{E}_{R}\left(\xi \mid \mathcal{F}_{s}\right), \quad 0 \leq s \leq t \leq T
$$

for an $\mathcal{F}$-measurable random variable $\xi$.

We denote by $F M_{t}$ the model of the financial market with $t$ periods defined on a probability space equipped with a filtration $\left(\Omega, \mathcal{F}_{0} \subset \cdots \subset \mathcal{F}_{t}, \mathrm{P}_{t}\right)$ and with price processes $\left(X_{0}^{k}, \ldots, X_{t}^{k}\right), k=1, \ldots, d$.

Let $t=0, \ldots, T-1$. Given an arbitrary atom $A$ of the $\sigma$-algebra $\mathcal{F}_{t}$ we define the $\sigma$-algebra $\mathcal{F}_{t+1}(A)=\left\{B \in \mathcal{F}_{t+1} \mid B \subset A\right\}$. Put

$$
R_{t+1}^{A}(B)=\frac{R(B)}{R(A)}
$$

for an arbitrary event $B \in \mathcal{F}_{t+1}(A)$.

Lemma 3.1. Let $t=0, \ldots, T-1$ and let $A_{1}, \ldots, A_{k}$ be all the atoms of the $\sigma$-algebra $\mathcal{F}_{t}$. Then

$$
R_{t+1}(B)=\sum_{i=1}^{k} R_{t}\left(A_{i}\right) R_{t+1}^{A_{i}}\left(A_{i} \cap B\right)
$$

for $B \in \mathcal{F}_{t+1}$.

Proof. The proof of the lemma follows from the definition of the probability measures $R_{t}$ and $R_{t+1}^{A}$.

Lemma 3.2. The following two statements hold.

1) $\mathrm{Q}$ is a martingale measure if and only if $\mathrm{Q}_{t}$ is a martingale measure for the model $F M_{t}$ of the financial market with $t$ periods for all $t=1, \ldots, T$.

2) $\mathrm{Q}$ is a minimal martingale measure if and only if $\mathrm{Q}_{t}$ a minimal martingale measure for the model $F M_{t}$ of the financial market with $t$ periods for all $t=1, \ldots, T$. 
Proof of statement 1). Sufficiency follows from the equality $\mathrm{Q}_{T}=\mathrm{Q}$.

Necessity. Let $\mathrm{Q}$ be a martingale measure for the model $F M_{T}$ of the financial market with $T$ periods. For $t=1, \ldots, T$, consider the measure $Q_{t}$ for the model $F M_{t}$ of the financial market with $t$ periods. We check that $\mathrm{Q}_{t}$ is a martingale measure for $F M_{t}$. Let $Y^{k}=\left(X_{0}^{k}, \ldots, X_{t}^{k}\right), k=1, \ldots, d$, be the prices up to the moment $t$. By the definition of the measure $\mathrm{Q}_{t}$, it is equivalent to the measure $\mathrm{P}_{t}$, since $\mathrm{Q}$ is equivalent to $\mathrm{P}$. Then equality (8) implies that $Y^{k}$ is a $\mathrm{Q}_{t}$-martingale.

Proof of statement 2). Sufficiency follows from the equality $\mathrm{Q}_{T}=\mathrm{Q}$.

Necessity. Let $\mathrm{Q}$ be a minimal martingale measure for the model $F M_{T}$ of the financial market with $T$ periods. For $t=1, \ldots, T$, consider the measure $\mathrm{Q}_{t}$ for the model $F M_{t}$ of the financial market with $t$ periods. Statement 1) implies that $\mathrm{Q}_{t}$ is a martingale measure.

Now we check that $\mathrm{Q}_{t}$ is a minimal martingale measure for the model $F M_{t}$. Let $M=\left(M_{0}, \ldots, M_{t}\right)$ be a $\mathrm{P}_{t}$-martingale defined on the probability space equipped with a filtration $\left(\Omega, \mathcal{F}_{0} \subset \cdots \subset \mathcal{F}_{t}, \mathrm{P}_{t}\right)$. Assume that $M$ is strongly $\mathrm{P}_{t^{-}}$orthogonal to $Y^{k}$, $k=1, \ldots, d$. We extend the stochastic process $M$ to the set $\{t+1, \ldots, T\}$ by putting $M_{t+1}=\cdots=M_{T}=M_{t}$. Then the stochastic process $N=\left(M_{0}, \ldots, M_{T}\right)$ is a Pmartingale.

Next we prove that the process $N$ is strongly P-orthogonal to the price processes $X^{k}$, $k=1, \ldots, d$. Let $k=1, \ldots, d$. Then

$$
\begin{aligned}
\mathrm{E}_{\mathrm{P}}\left(\left(M_{s+1}-M_{s}\right)\left(X_{s+1}^{k}-X_{s}^{k}\right) \mid \mathcal{F}_{s}\right) \\
\quad=\left\{\begin{array}{r}
\mathrm{E}_{\mathrm{P}_{t}}\left(\left(M_{s+1}-M_{s}\right)\left(X_{s+1}^{k}-X_{s}^{k}\right) \mid \mathcal{F}_{s}\right)=0, \\
\text { for } s<t, \text { since } M \perp Y^{k}, k=1, \ldots, d ; \\
0, \quad \text { for } s \geq t, \text { since } M_{s}=M_{s+1} \text { by definition. }
\end{array}\right.
\end{aligned}
$$

Thus $N$ is a $\mathrm{P}$-martingale that is strongly $\mathrm{P}$-orthogonal to the price processes $X_{1}, \ldots, X_{d}$. Since $\mathrm{Q}$ is a minimal martingale measure, $N$ is a Q-martingale. Lemma 3.3 implies that $M$ is a $Q_{t}$-martingale.

Therefore $\mathrm{Q}_{t}$ is a minimal martingale measure.

Therefore the probability measure $\mathrm{Q}_{t+1}, t=0, \ldots, T-1$, is uniquely determined by the measure $\mathrm{Q}_{t}$ and the family of conditional probability measures

$$
\left\{\mathrm{Q}_{t+1}^{A} \mid A \text { is an atom of the } \sigma \text {-algebra } \mathcal{F}_{t}\right\} \text {. }
$$

Given an arbitrary atom $A$ of the $\sigma$-algebra $\mathcal{F}_{t}$, consider the probability space

$$
\mathrm{P} S_{t+1}^{A}=\left(A, \mathcal{F}_{t+1}(A), \mathrm{P}_{t+1}^{A}\right) \text {. }
$$

Let $Y_{t}^{i A}=\left.\left(X_{t+1}^{i}-X_{t}^{i}\right)\right|_{A}, i=1, \ldots, d$, be the gain processes.

For every atom of the $\sigma$-algebra $\mathcal{F}_{t}$, we have a one-period model of the financial market defined on the probability space $\left(A, \mathcal{F}_{t}(A), \mathrm{P}_{A}\right)$ for which $Y_{t}^{1 A}, \ldots, Y_{t}^{d A}$ are the gain vectors. We denote these one-period models of the financial market by $F M_{t}^{A}$.

The indicator function of an arbitrary set $A \in \mathcal{F}$ is denoted by $\chi_{A}$.

Lemma 3.3. Let $t=0, \ldots, T-1$. Then $\mathrm{Q}_{t+1}$ is a martingale measure for the model $F M_{t+1}$ of the financial market with $t+1$ periods if and only if

(i) $\mathrm{Q}_{t}$ is a martingale measure for the model $F M_{t}$ of the financial market with $t$ periods, and

(ii) $\mathrm{Q}_{t+1}^{A}$ is a martingale measure for the one-period model $F M_{t}^{A}$ of the financial market, where $A$ is an arbitrary atom of the $\sigma$-algebra $\mathcal{F}_{t}$. 
Proof. Since $\mathrm{P}$ and $\mathrm{Q}$ are equivalent probability measures, $\mathrm{P}_{t}$ and $\mathrm{Q}_{t}$ are equivalent probability measures for all $t \in \mathbb{T}$.

Necessity. Let $\mathrm{Q}_{t+1}$ be a martingale measure. Then Lemma 3.2 implies that $\mathrm{Q}_{t}$ is a martingale measure for the model $F M_{t+1}$. Let $A$ be an atom of the $\sigma$-algebra $\mathcal{F}_{t}$. First we prove that $\mathrm{Q}_{t+1}^{A}$ is a martingale measure. For all $k=1, \ldots, d$, we have

$$
\mathrm{E}_{Q_{t+1}^{A}} X_{t+1}^{k}=\frac{\mathrm{E}_{\mathrm{Q}_{t+1}}\left(X_{t+1}^{k} \chi_{A}\right)}{\mathrm{Q}_{t}(A)}=\frac{\mathrm{E}_{\mathrm{Q}_{t+1}}\left(X_{t}^{k} \chi_{A}\right)}{\mathrm{Q}_{t}(A)}=\frac{\mathrm{E}_{\mathrm{Q}_{t}}\left(X_{t}^{k} \chi_{A}\right)}{\mathrm{Q}_{t}(A)}=\left.X_{t}^{k}\right|_{A}
$$

The second equality above holds in view of the property that $Q_{t+1}$ is a martingale measure, while the last equality holds, since the random variable $X_{t}^{k}$ is constant on every atom of the $\sigma$-algebra $\mathcal{F}_{t}$.

Sufficiency. Let the probability measure $Q_{t}$ be a martingale measure for the model $F M_{t}$ and let $\mathrm{Q}_{t}$ be martingale measures for all models $F M_{t}^{A}$, where $A$ denotes an arbitrary atom of the $\sigma$-algebra $\mathcal{F}_{t}$. Since $Q_{t}$ is a martingale measure for the model $F M_{t}$, equality (8) implies that

$$
\mathrm{E}_{Q_{t+1}}\left(X_{s+1}^{k} \mid \mathcal{F}_{s}\right)=X_{s}^{k}
$$

for all $k=0, \ldots, d$ and $s=0, \ldots, t-1$.

It remains to show that $\mathrm{E}_{\mathrm{Q}_{t+1}}\left(X_{t+1} \mid \mathcal{F}_{t}\right)=X_{t}$. Given an arbitrary atom $A$ of the $\sigma$-algebra $F_{t}$, we have

$$
\mathrm{E}_{\mathrm{Q}_{t+1}}\left(X_{t+1}^{k}-X_{t}^{k}\right) \chi_{A}=\mathrm{Q}_{t}(A) \mathrm{E}_{\mathrm{Q}_{t+1}^{A}} Y_{t}^{k A}=0
$$

where the last equality holds, since $\mathrm{Q}_{t}^{A}$ is a martingale measure for the model $F M_{t}^{A}$.

Lemma 3.4. Let $t=0, \ldots, T-1$. Then $\mathrm{Q}_{t+1}$ is a minimal martingale measure for the model $F M_{t+1}$ with $t+1$ periods if and only if

(i) $Q_{t}$ is a minimal martingale measure for the model $F M_{t}$ with $t$ periods, and

(ii) $\mathrm{Q}_{t+1}^{A}$ is a minimal martingale measure for the one-period model $F M_{t}^{A}$ for all atoms $A$ of the $\sigma$-algebra $\mathcal{F}_{t}$.

Proof. Necessity. Let $\mathrm{Q}_{t+1}$ be a minimal martingale measure for the model $F M_{t+1}$. Lemma 3.3 implies that $\mathrm{Q}_{t}$ is a martingale measure for the model $F M_{t}$ and that $\mathrm{Q}_{t}^{A}$ is a martingale measure for the model $F M_{t}^{A}$ for all atoms of the $\sigma$-algebra $\mathcal{F}_{t}$.

Let $M=\left(M_{0}, \ldots, M_{t}\right)$ be an arbitrary $\mathrm{P}_{t}$-martingale that is strongly $\mathrm{P}_{t}$-orthogonal to the price processes $\left(X_{0}^{k}, \ldots, X_{t}^{k}\right), k=1, \ldots, d$. We extend the process $M$ by putting $M_{t+1}=M_{t}$. The resulting process $\widetilde{M}=\left(M_{0}, \ldots, M_{t+1}\right)$ is a $\mathrm{P}_{t+1}$-martingale. Note that the process $\widetilde{M}$ is strongly $\mathrm{P}_{t+1}$-orthogonal to $\left(X_{0}^{k}, \ldots, X_{t+1}^{k}\right), k=1, \ldots, d$. Since $\mathrm{Q}_{t+1}$ is a minimal martingale measure, we derive that $\widetilde{M}$ is a $\mathrm{Q}_{t+1}$-martingale. Then equality (8) implies that $M$ is a $\mathrm{Q}_{t}$-martingale. Therefore $\mathrm{Q}_{t}$ is a minimal martingale measure for the model $F M_{t}$.

Let $M=\left(M_{0}, M_{1}\right)$ be an arbitrary $\mathrm{P}_{t+1}^{A}$-martingale for the model $F M_{t+1}^{A}$. Assume that $M$ is orthogonal to the price processes and introduce another stochastic process $N=\left(N_{0}, \ldots, N_{t+1}\right)$, where $N_{0}=\cdots=N_{t}=0$ and $N_{t+1}=M_{1}-M_{0}$. Then $N$ is a $\mathrm{P}_{t+1}$-martingale that is orthogonal to the price processes, whence we conclude that it is a $\mathrm{Q}_{t+1}$-martingale. Thus $M$ is a $\mathrm{Q}_{t+1}^{A}$-martingale for the model $F M_{t+1}^{A}$. Therefore $\mathrm{Q}_{t+1}^{A}$ is a minimal martingale measure for the model $F M_{t+1}^{A}$.

Sufficiency. Let $\mathrm{Q}_{t}$ be a minimal martingale measure for the model $F M_{t}$ and let $\mathrm{Q}_{t+1}^{A}$ be a minimal martingale measure for the model $F M_{t}^{A}$ and for all atoms $A_{1}, \ldots, A_{k}$ of the $\sigma$-algebra $\mathcal{F}_{t}, k>0$. We check that $\mathrm{Q}_{t+1}$ is a minimal martingale measure for the model $F M_{t+1}$. It follows from Lemma 3.3 that $\mathrm{Q}_{t+1}$ is a martingale measure. 
Let $M=\left(M_{0}, \ldots, M_{t+1}\right)$ be an arbitrary $\mathrm{P}_{t+1}$-martingale that is strongly $\mathrm{P}_{t+1^{-}}$-orthogonal to the price processes $\left(X_{0}^{k}, \ldots, X_{t+1}^{k}\right), k=1, \ldots, d$. Let $N=\left(M_{0}, \ldots, M_{t}\right)$. The stochastic process $N$ is a $\mathrm{P}_{t}$-martingale that is strongly $\mathrm{P}_{t}$-orthogonal to the price processes $\left(X_{0}^{k}, \ldots, X_{t}^{k}\right), k=1, \ldots, d$. Since $\mathrm{Q}_{t}$ is a minimal orthogonal measure for the model $F M_{t}$, the process $N$ is a $\mathrm{Q}_{t}$-martingale. Using equality (8), we obtain

$$
0=\mathrm{E}_{\mathrm{Q}_{t}}\left(M_{s+1}-M_{s} \mid \mathcal{F}_{s}\right)=\mathrm{E}_{Q_{t+1}}\left(M_{s+1}-M_{s} \mid \mathcal{F}_{s}\right)
$$

for all $s=0, \ldots, t-1$.

Let $A$ be an arbitrary atom of the $\sigma$-algebra $\mathcal{F}_{t}$. The process $N_{A}=\left(\left.M_{t}\right|_{A},\left.M_{t+1}\right|_{A}\right)$ is a $\mathrm{P}_{t}^{A}$-martingale for the model $F M_{t}^{A}$ that is strongly $\mathrm{P}_{t}^{A}$-orthogonal to the price processes. Since $\mathrm{P}_{t}^{A}$ is minimal for the model $F M_{t}^{A}$, we conclude that $N_{A}$ is a $\mathrm{Q}_{t}^{A}$ martingale for $\mathrm{P} S_{t}^{A}$. Then

$$
\mathrm{E}_{\mathrm{Q}_{t+1}}\left(M_{t+1}-M_{t}\right) \chi_{A}=\frac{\mathrm{E}_{\mathrm{Q}_{t+1}^{A}}\left(M_{t+1}-M_{t}\right)}{\mathrm{Q}_{t}(A)}=0 .
$$

Equalities (9) and (10) imply that $M$ is a $Q_{t+1}$-martingale. Thus $Q_{t+1}$ is a minimal martingale measure for the model $F M_{t+1}$.

Theorem 3.1. 1. There exists at most one minimal martingale measure.

2. A minimal martingale measure exists if and only if, for all $t=0, \ldots, T-1$ and for an arbitrary atom $A$ of the $\sigma$-algebra $\mathcal{F}_{t}$, there exists a minimal martingale measure for the one-period model $F M_{t}^{A}$.

Proof. Since there exists at most one minimal martingale measure for the one-period models $F M_{t}^{A}$ for all $t=0, \ldots, T-1$ and an arbitrary atom of the $\sigma$-algebra $\mathcal{F}_{t}$, the proof of statement 1 follows.

The proof of statement 2 follows from Lemmas 3.1 3.4

Corollary 3.1 (A procedure to construct a minimal martingale measure). The procedure consists of the following five steps.

Step 1 Set $t=0$ and define the initial measure $\mathrm{Q}_{0}$ by putting

$$
\mathrm{Q}_{0}(\varnothing)=0 \text { and } \mathrm{Q}_{0}(\Omega)=1 .
$$

Step 2 The measure $\mathrm{Q}_{t}$ is defined. For an arbitrary atom $A$ of the $\sigma$-algebra $\mathcal{F}_{t}$, consider the one-period model $F M_{t}^{A}$. Using equalities (4), (5), and (17) evaluate the numbers $r_{1}, \ldots, r_{d}$. If all these numbers are of the same sign, then the minimal martingale measure exists for the model $F M_{t}^{A}$ and is given by

$$
\mathrm{Q}_{t+1}^{A}\left(\left\{\omega_{i}\right\}\right)=\frac{r_{i}}{\sum_{k=1}^{d} r_{i}}, \quad i=1, \ldots, N .
$$

Otherwise, if not all numbers $r_{1}, \ldots, r_{d}$ have the same sign, then there is no minimal martingale measure for the model $F M_{t}^{A}$.

Step 3 If a minimal martingale measure does not exist in the one-period model $F M_{t}^{A}$ for at least one atom $A$ of the $\sigma$-algebra $\mathcal{F}_{t}$, then the minimal martingale measure does not exist and the procedure stops.

Step 4 Applying Lemma 3.1 we evaluate the measure $\mathrm{Q}_{t+1}$ as follows. Let $t=0, \ldots, T-1$ and let $A_{1}, \ldots, A_{k}$ be all the atoms of the $\sigma$-algebra $\mathcal{F}_{t}$. For $B \in \mathcal{F}_{t+1}$, we put

$$
\mathrm{Q}_{t+1}(B):=\sum_{i=1}^{k} \mathrm{Q}_{t}\left(A_{i}\right) \mathrm{Q}_{t+1}^{A}\left(A_{i} \cap B\right) .
$$

Step 5 Increase $t$ by one. If $t=T$, then the minimal martingale measure $\mathrm{Q}=\mathrm{Q}_{T}$ is found and the procedure stops; otherwise go to Step 2. 


\section{EXamples of the eValuation of a minimal martingale measure}

4.1. A one-period model of the financial market with two risky assets. Let $N>0, \Omega=\left(\omega_{1}, \ldots, \omega_{N}\right), \mathrm{P}\left(\omega_{i}\right)=p_{i}, i=1, \ldots, N$, and

$$
Y^{i}\left(\omega_{j}\right)=y_{i j}, \quad i=1,2, j=1, \ldots, N .
$$

By $Q$ we denote the minimal martingale measure to be evaluated,

$$
\mathrm{Q}\left(\omega_{i}\right)=q_{i}, \quad i=1, \ldots, N .
$$

The numbers $r_{1}, \ldots, r_{N}$ are evaluated according to Theorem 2.1, Let

$$
\Delta=\left|\begin{array}{ll}
\mathrm{E}\left(Y^{1}\right)^{2} & \mathrm{E} Y^{1} Y^{2} \\
\mathrm{E} Y^{1} Y^{2} & \mathrm{E}\left(Y^{2}\right)^{2}
\end{array}\right|, \quad \Delta_{1}=\left|\begin{array}{ll}
\mathrm{E} Y^{1} & \mathrm{E} Y^{1} Y^{2} \\
\mathrm{E} Y^{2} & \mathrm{E}\left(Y^{2}\right)^{2}
\end{array}\right|, \quad \Delta_{2}=\left|\begin{array}{ll}
\mathrm{E}\left(Y^{1}\right)^{2} & \mathrm{E} Y^{1} \\
\mathrm{E} Y^{1} Y^{2} & \mathrm{E} Y^{2}
\end{array}\right| .
$$

Then we have

$$
r_{i}=\Delta-\Delta_{1} y_{1 i}-\Delta_{2} y_{2 i}, \quad i=1, \ldots, N .
$$

If all numbers $r_{i}$ are of the same sign, then the minimal martingale measure exists and the $q_{i}$ are proportional to the $r_{i}$ (see Theorem 2.1). Otherwise the minimal martingale measure does not exist.

Below are two numerical examples of the evaluation of a minimal martingale measure.

Example 4.1. Let $N=3$ and let

$$
\begin{gathered}
\mathrm{P}\left(\omega_{1}\right)=\frac{1}{6}, \quad \mathrm{P}\left(\omega_{2}\right)=\frac{1}{3}, \quad \mathrm{P}\left(\omega_{3}\right)=\frac{1}{2}, \\
Y^{1}\left(\omega_{1}\right)=Y^{1}\left(\omega_{2}\right)=1, \quad Y^{1}\left(\omega_{3}\right)=-1, \\
Y^{2}\left(\omega_{1}\right)=1, \quad Y^{2}\left(\omega_{2}\right)=-2, \quad Y^{2}\left(\omega_{3}\right)=1 .
\end{gathered}
$$

Using equalities (11) we get

$$
r_{1}=r_{2}=r_{3}=1 \text {. }
$$

Since the numbers $r_{1}, r_{2}$, and $r_{3}$ are of the same sign, the minimal martingale measure exists and its values are proportional to $r_{1}, r_{2}$, and $r_{3}$. Therefore

$$
q_{1}=q_{2}=q_{2}=\frac{1}{3}
$$

Example 4.2. Let $N=3$ and let

$$
\begin{gathered}
\mathrm{P}\left(\omega_{1}\right)=\frac{1}{6}, \quad \mathrm{P}\left(\omega_{2}\right)=\frac{1}{3}, \quad \mathrm{P}\left(\omega_{3}\right)=\frac{1}{2}, \\
Y^{1}\left(\omega_{1}\right)=Y^{1}\left(\omega_{2}\right)=1, \quad Y^{1}\left(\omega_{3}\right)=-1, \\
Y^{2}\left(\omega_{1}\right)=-3, \quad Y^{2}\left(\omega_{2}\right)=-2, \quad Y^{2}\left(\omega_{3}\right)=1 .
\end{gathered}
$$

Using equalities (11) we get

$$
r_{1}=-\frac{1}{3}, \quad r_{2}=\frac{1}{3}, \quad r_{3}=\frac{1}{9} .
$$

Since not all numbers $r_{1}, r_{2}, r_{3}$ have the same sign, the minimal martingale measure does not exist. 
4.2. A two-period model of the financial market with two risky assets. Let $\Omega=\left\{\omega_{i j} \mid i, j=1,2,3\right\}, p_{1}=\frac{1}{6}, p_{2}=\frac{1}{3}, p_{3}=\frac{1}{2}$,

$$
\mathcal{F}_{0}=\{\varnothing, \Omega\}, \quad \mathcal{F}_{1}=\sigma a\left(\left\{A_{i} \mid i=1,2,3\right\}\right), \quad \mathcal{F}_{2}=2^{\Omega},
$$

where $A_{i}=\left\{\omega_{i j} \mid j=1,2,3\right\}, i=1,2,3$, and $\mathrm{P}\left(\omega_{i j}\right)=p_{i} p_{j}, i, j=1,2,3$.

Let $a_{1}=a_{2}=1, a_{3}=-1$ and $b_{1}=1, b_{2}=-2, b_{3}=1$. Put

$$
X_{1}^{1}\left(w_{i j}\right)=1+a_{i}, \quad X_{2}^{1}\left(w_{i j}\right)=1+a_{i}+a_{j}, \quad i, j=1,2,3,
$$

and

$$
X_{1}^{2}\left(w_{i j}\right)=1+b_{i}, \quad X_{2}^{2}\left(w_{i j}\right)=1+b_{i}+b_{j}, \quad i, j=1,2,3 .
$$

Following the procedure of Corollary 3.1, we first find the measure $Q_{1}$. In fact, the measure $Q_{1}$ is found in Example 4.1. Thus

$$
\mathrm{Q}_{1}\left(A_{1}\right)=\mathrm{Q}_{1}\left(A_{2}\right)=\mathrm{Q}_{1}\left(A_{3}\right)=\frac{1}{3} .
$$

Then we evaluate the measure $\mathrm{Q}_{2}$. For this, we need to evaluate the measures $\mathrm{Q}_{2}^{A_{1}}, \mathrm{Q}_{2}^{A_{2}}$, and $\mathrm{Q}_{2}^{A_{3}}$. The problems of the evaluation of the measures $\mathrm{Q}_{2}^{A_{1}}, \mathrm{Q}_{2}^{A_{2}}$, and $\mathrm{Q}_{2}^{A_{3}}$ coincide with the problem solved in Example 4.1. Thus

$$
\mathrm{Q}^{A_{i}}\left(\omega_{i j}\right)=\frac{1}{3}, \quad i, j=1,2,3 .
$$

Then we find from Lemma 3.1 that

$$
\mathrm{Q}_{2}\left(\omega_{i j}\right)=\frac{1}{27}, \quad i, j=1,2,3 .
$$

\section{BIBLIOGRAPHY}

1. M. Schweizer, Hedging of Options in a General Semimartingale Model, Diss. ETH Zurich 8615, 1988 .

2. M. Schweizer, Option hedging for semimartingales, Stoch. Process. Appl. 37 (1991), 339-363. MR.1102880 (92c:90025)

3. H. Föllmer and M. Schweizer, Hedging of contingent claims under incomplete information, Applied Stochastic Analysis (M. H. A. Davis and R. J. Elliott, eds.), Stochastics Monographs, vol. 5, Gordon and Breach, New York, 1991, pp. 389-414. MR.1108430 (92g:90029)

4. M. Schweizer, On the minimal martingale measure and the Föllmer-Schweizer decomposition, Stoch. Anal. Appl. 13 (1995), 573-599. MR.1353193 (96h:60080)

5. T. Arai, The relations between minimal martingale measure and minimal entropy martingale measure, Asia-Pacific Financial Markets 8 (2001), 137-177.

6. F. Biagini and M. Pratelli, Local risk minimization and numéraire, J. Appl. Probab. 36 (1999), 1126-1139. MR1742155(2001e:91079)

7. T. Choulli and C. Stricker, Minimal entropy-Hellinger martingale measure in incomplete markets, Math. Finance 15 (2005), 465-490. MR2147158 (2006f:91065)

8. A. Cerny and J. Kallsen, On the structure of general mean-variance hedging strategies, Ann. Probab. 35 (2007), 1479-1531. MR2330978 (2009e:60154)

9. T. Chan, Pricing contingent claims on stocks driven by Lévy processes, Ann. Appl. Probab. 9 (1999), 504-528. MR 1687394 (2000f:91028)

10. D. B. Colwell and R. J. Elliott, Discontinuous asset prices and non-attainable contingent claims, Math. Finance 3 (1993), 295-308.

11. H. Föllmer and A. Schied, Stochastic Finance: An Introduction in Discrete Time, Walter de Gruyter, 2004. MR2169807 (2006d:91002)

Faculty for Mechanics and Mathematics, National Taras Shevchenko University, AcademiCian Glushkov Avenue 4E, Kyiv 03127, Ukraine

E-mail address: vadym.doroshenko@gmail.com

Received 22/JAN/2011

Translated by N. SEMENOV 